\title{
Analysis of Atmospheric Transmission Characteristics in MIR Spectrum of VIIRS
}

\author{
Yanbiao Sun ${ }^{1, ~ a}$, Xingbang $\mathrm{Hu}^{2, \text { b }}$, Liqin $\mathrm{He}^{2, c}$, Shuaiyang Zhao ${ }^{2, \mathrm{~d}}$, Lei Yan ${ }^{1, \mathrm{e}^{*}}$ \\ ${ }^{1}$ Chinese Academy of Surveying \& Mapping, Beijing 100039, China \\ ${ }^{2}$ Institute of Remote Sensing \&Geographical Information System, Peking University, Beijing 100871, \\ China \\ asyb51@163.com, bxingbang_hu@163.com, cheliqin_de@126.com, dsyzhao@pku.edu.cn \\ e*lyan@pku.edu.cn
}

\begin{abstract}
Keywords: Middle infrared; MODTRAN; Atmospheric transmittance; VIIRS
Abstract. The atmospheric transmission is an important parameter affecting the solar radiation and surface thermal radiation. In this paper, MODTRAN radiative transfer model was used to analysis the atmospheric Transmission in middle infrared (MIR) spectrum of VIIRS. We have quantitatively simulated and analyzed the effect of atmospheric profile, aerosol type, visibility, moisture content and observed zenith angle on atmospheric transmittance using the two MIR channels of VIIRS (M12 band and M13-band). The results showed that the transmissivities of the both two middle infrared channels M12 and M13 of VIIRS are influenced by the aerosol type, visibility and observation angle. And the influence of these factors on the M12 channel transmittance are greater impact, in addition, M12 band is strongly influenced by the change of atmospheric profile.
\end{abstract}

\section{Introduction}

Atmospheric transmittance is an important parameter for the radiation of solar and surface, which is also significant in inverting the surface parameters, such as surface temperature, surface reflectivity, etc. When the solar radiation and surface radiation are transport in the atmosphere, they are not only affected by the absorption and scattering of atmospheric molecules such as $\mathrm{H}_{2} \mathrm{O}$, mixed gases $\left(\mathrm{CO}_{2}\right.$, $\left.\mathrm{CO}, \mathrm{N}_{2} \mathrm{O}, \mathrm{CH}_{4}, \mathrm{O}_{2}\right)[1], \mathrm{O}_{3}, \mathrm{~N}_{2}$, but also affected by aerosol particles, that will result the radiation attenuation in solar and surface. The medium infrared band is between the visible-near-infrared (VIR) and thermal infrared (IR) bands, so its energy exhibits a unique transition in the transmission process, and its atmospheric transmission characteristics are significantly changed. On the other hand, since the remote sensing load is a band-pass system, the actual energy value measured by the load is greatly affected by the atmospheric transmittance of channels[2]. Therefore, it is necessary to carry out an in-depth analysis on the total atmospheric transmittance and the influence factors of channel transmittance.

Bohui Tang, Zhaoliang Li et al. have analyzed the influence factors of transmittance of the medium-wave infrared channels $22(3.929-3.989 \mu \mathrm{m})$ and $23(4.020-4.080 \mu \mathrm{m})$ of MODIS [3]. But their research were mainly focused on the analysis of aerosol and water vapor content, and did not considered the other influence factors of atmospheric transmittance. In addition, their research were only analyzed the required 22 and 23 channels, ignoring other medium-wave infrared channels. Zhiguo Rong[4], Yonggang Qian[5] have briefly analyzed the characteristics of atmospheric radiation of the medium-wave infrared channel, and did not analyze the transmittance of the medium-wave infrared channel.

In the visible-near-infrared band and the thermal infrared band, there are many literatures on atmospheric transmittance and the study are also mature[6]. However, the atmosphere spectral transmittance and channel transmittance of middle infrared band have not analyzed in detail in the present paper. In this paper, we have use MODTRAN4 radiation correction model[7] to analyze the atmospheric transmittance of the middle wave infrared channels. On this basis, we have 
quantitatively simulated and analyzed the effect of atmospheric profile, aerosol type, visibility, moisture content and observed zenith angle on atmospheric transmittance using the two middle infrared channels of VIIRS (M12 band, 3.660-3.840 $\mu \mathrm{m}$, center wavelength 3.697 $\mu \mathrm{m}$ and M13-band, 3.973-4.128 $\mu \mathrm{m}$, center wavelength $4.05 \mu \mathrm{m})$.

\section{The VIIRS Instrument}

The Visible/Infrared Imager Radiometer Suite (VIIRS)[8] is one of the five major Earth observing instruments onboard S-NPP and JPSS. VIIRS signifies a new era of moderate-resolution imaging capabilities following the legacy of AVHRR and Moderate-Resolution Imaging Spectroradiometer (MODIS).

The VIIRS instrument is a whiskbroom scanning radiometer with a field of regard of $112.6^{\circ}$ in the cross-track direction. At a nominal altitude of $824 \mathrm{~km}$, the swath width is $3040 \mathrm{~km}$, providing full daily coverage both in the day and night side of the Earth. VIIRS has 22 spectral bands covering the spectrum between $0.412 \mu \mathrm{m}$ and $12.01 \mu \mathrm{m}$, including 16 moderate resolution bands (M-bands) with a spatial resolution of $750 \mathrm{~m}$ at nadir, 5 imaging resolution bands (I-bands) - $375 \mathrm{~m}$ at nadir, and one panchromatic daynight band (DNB) with a $750 \mathrm{~m}$ spatial resolution throughout the scan. The M-bands include 11 reflective solar bands (RSB) and 5thermal emissive bands (TEB). The I-bands include 3 RSB bands and 2 TEB bands (Tables 1) [9].

Table 1. VIIRS Band Centers, Spatial Resolution, and Gain

\begin{tabular}{|c|c|c|c|c|c|c|c|c|c|}
\hline & $\begin{array}{l}\text { Band } \\
\text { No. }\end{array}$ & Dr tving EDR(B\} & $\begin{array}{c}\text { Spec1ral } \\
\text { Aarge } \\
\text { \{un? }\end{array}$ & $\begin{array}{c}\text { Hortz Sample } \\
\text { (1rack } \\
\text { Nadir }\end{array}$ & $\begin{array}{l}\text { Inierval }(\mathrm{km}\} \\
\times \text { Soan\} } \\
\text { End of Scan }\end{array}$ & $\begin{array}{l}\text { Band } \\
\text { Galn }\end{array}$ & $\begin{array}{l}\text { Lyyp of } \\
\text { Tlyp } \\
\text { (Spec) }\end{array}$ & $\begin{array}{l}\text { Lmax or } \\
T \max \end{array}$ & $\begin{array}{l}\text { SNR o } \\
\text { NEdT }\langle K\}\end{array}$ \\
\hline \multirow{14}{*}{ 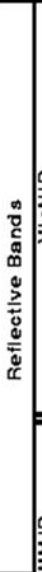 } & M1 & $\begin{array}{c}\text { Ocean Colot } \\
\text { Aerosol }\end{array}$ & $0.402-0.422$ & $0.742 \times 0.253$ & $1.80 \times 1.58$ & $\begin{array}{l}\text { Hlgh } \\
\text { Law }\end{array}$ & $\begin{array}{l}449 \\
155 \\
\end{array}$ & $\begin{array}{l}135 \\
815 \\
\end{array}$ & $\begin{array}{l}352 \\
318 \\
\end{array}$ \\
\hline & M2 & $\begin{array}{c}\text { Ocean Colot } \\
\text { Aetorol }\end{array}$ & $0.439-0.454$ & $0.742 \times 0.253$ & $1.80 \times 1.59$ & $\begin{array}{l}\text { Hlgh } \\
\text { Law }\end{array}$ & $\begin{array}{l}40 \\
148\end{array}$ & $\begin{array}{l}127 \\
997\end{array}$ & $\begin{array}{l}380 \\
409\end{array}$ \\
\hline & мз & $\begin{array}{c}\text { Ocean Color } \\
\text { Aerosol }\end{array}$ & $0.479-0.498$ & $0.742 \times 0.259$ & $1.80 \times 1.58$ & $\begin{array}{l}\text { High } \\
\text { Low }\end{array}$ & $\begin{array}{c}32 \\
123\end{array}$ & $\begin{array}{l}107 \\
702 \\
\end{array}$ & $\begin{array}{l}410 \\
414\end{array}$ \\
\hline & $\mathrm{Mu}_{4}$ & $\begin{array}{c}\text { Ocean Color } \\
\text { Aetosol }\end{array}$ & $0.545-0.595$ & $0.742 \times 0.259$ & $1.80 \times 1.59$ & $\begin{array}{l}\text { High } \\
\text { Low }\end{array}$ & $\begin{array}{l}21 \\
90 \\
\end{array}$ & $\begin{array}{c}79 \\
697 \\
\end{array}$ & $\begin{array}{l}382 \\
315 \\
\end{array}$ \\
\hline & $\pi$ & Imagery EOR & $0.800-0.880$ & $0.371 \times 0.397$ & $0.80 \times 0.793$ & Slagle & 22 & 718 & 119 \\
\hline & M5 & $\begin{array}{c}\text { Ocean Color } \\
\text { Aetosol }\end{array}$ & $0.882-0.892$ & $0.742 \times 0.259$ & $1.80 \times 1.58$ & $\begin{array}{l}\text { High } \\
\text { Low }\end{array}$ & $\begin{array}{l}10 \\
89 \\
\end{array}$ & $\begin{array}{r}53 \\
851 \\
\end{array}$ & $\begin{array}{l}242 \\
380 \\
\end{array}$ \\
\hline & Mg & Atmosph. Correct. & $0.739-0.754$ & $0.722 \times 0.779$ & $1.80 \times 1.58$ & Slngle & 3.8 & 41 & 139 \\
\hline & 2 & NOVI & $0.848-0.895$ & $0.371 \times 0.337$ & $0.80 \times 0.793$ & Slngle & 25 & 343 & 150 \\
\hline & $M 7$ & $\begin{array}{c}\text { Ocean Colot } \\
\text { Aerosol }\end{array}$ & $0.940-0.995$ & $0.742 \times 0.253$ & $1.80 \times 1.58$ & $\begin{array}{l}\text { High } \\
\text { Low }\end{array}$ & $\begin{array}{r}8.4 \\
33.4 \\
\end{array}$ & $\begin{array}{r}29 \\
349 \\
\end{array}$ & $\begin{array}{l}215 \\
340 \\
\end{array}$ \\
\hline & м9 & Cloud Partble Slze & $1230-1250$ & $0.722 \times 0.770$ & $1.80 \times 1.59$ & Slogle & 5.4 & 185 & 74 \\
\hline & M9 & Clerlua:Clo ud Cover & $1.371-1.398$ & $0.742 \times 0.778$ & $1.80 \times 1.59$ & Slogle & 8 & $\pi .1$ & 93 \\
\hline & 13 & Blpary Snow $\mathrm{M}$ ap & $1,590-1.840$ & $0.371 \times 0.337$ & $0.80 \times 0.799$ & Slogle & 7.3 & 72.5 & 8 \\
\hline & M10 & Srow Fraction & $1.580-1.840$ & $0.742 \times 0.778$ & $1.80 \times 1.58$ & Slngle & 7.3 & 71.2 & 342 \\
\hline & M11 & Clouda & $2225-2275$ & $0.742 \times 0.779$ & $1.80 \times 1.59$ & Slngle & 0.12 & 31.9 & 10 \\
\hline \multirow{7}{*}{ 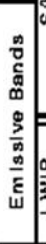 } & $\mathrm{A}$ & Imagery Clouxda & $3.550-3.930$ & $0.371 \times 0.397$ & $0.80 \times 0.799$ & Slogie & 270 & 353 & 2.5 \\
\hline & $M 12$ & SST & $3.800-3.840$ & $0.742 \times 0.770$ & $1.80 \times 1.58$ & Slagle & 270 & 353 & 0.390 \\
\hline & $\mathrm{M} 13$ & $\begin{array}{c}\text { SST } \\
\text { Flre日 }\end{array}$ & $3973-4.128$ & $0.742 \times 0.259$ & $1.80 \times 1.58$ & $\begin{array}{l}\text { Hlgh } \\
\text { Low }\end{array}$ & $\begin{array}{l}300 \\
390 \\
\end{array}$ & $\begin{array}{l}343 \\
034\end{array}$ & $\begin{array}{l}0.107 \\
0.423 \\
\end{array}$ \\
\hline & W14 & Cloud Top Propertles & $8.400-8.700$ & $0.722 \times 0.778$ & $1.80 \times 1.59$ & Slngle & 270 & 330 & 0.091 \\
\hline & W15 & SST & $10.283-11203$ & $0.722 \times 0.770$ & $1.80 \times 1.59$ & Slngib & 300 & 343 & 0.07 \\
\hline & 5 & Cloud Imagery & $10.500-12.400$ & $0.371 \times 0.337$ & $0.80 \times 0.793$ & Slngle & 210 & 340 & 1.5 \\
\hline & M16 & SST & $11,539-12,498$ & $0.720 \times 0.770$ & $1.80 \times 1.59$ & Slngite & 300 & 340 & 0.072 \\
\hline
\end{tabular}

\section{Simulation and analysis of VIIRS MIR Channel transmittance}

MODTRAN4(MODeratespectral resolution atmospheric TRANsmittance algorithm and computer model), the newly released version of the U.S. Air Force atmospheric transmission, radiance and flux model is being developed jointly by the Air Force Research Laboratory/Space Vehicles Directorate and Spectral Sciences, Inc. It is expected to provide the accuracy required for analyzing spectral data for both atmospheric and surface characterization.

VIIRS data has 7 TIR channels $(3.5 \sim 14.5 \mu \mathrm{m})$ among all of its 22 channels. They are channels M12-M16 and channels I4-I5. Therefore, selecting appropriate TIR bands by conditional analysis of atmospheric window is necessary. 
Figure1 shows atmospheric transmissions curve at viewing angle $45^{\circ}$ from nadir in mid-latitude summer condition(column water vapor, $\mathrm{cwv}=2.9 \mathrm{~cm}$; surface visibility at $0.55 \mu \mathrm{m}$, vis. $=23 \mathrm{~km}$ ). We can see from figure 1 that there are two atmospheric windows in the spectral scope of $3.5 \sim 14.5$ $\mu \mathrm{m}$. One is $8 \sim 13 \mu \mathrm{m}$ atmospheric window and the other is $3.5 \sim 4.2 \mu \mathrm{m}$ atmospheric window.

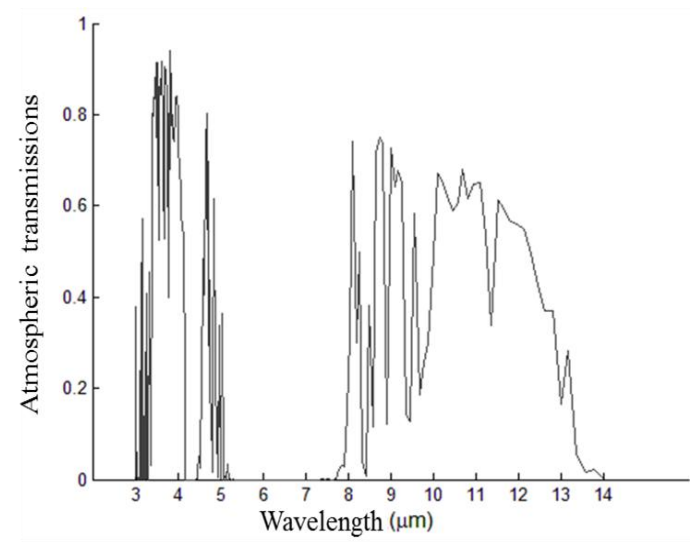

Fig.1 Atmospheric transmissions curve

In this paper, the M12 and M13 channels of VIIRS are taken as an example to analyze the influence factors of the transmittance of medium infrared channel in 3.5-4.2 $\mu \mathrm{m}$. This paper will quantitatively analyze for which atmospheric components or which atmospheric conditions that the transmittance of these two bands will sensitive in the atmospheric correction. First, we use MODTRAN4 to quantitatively analyze the two-channel transmittance on different atmospheric profiles (tropical, mid-latitude summer, mid-latitude winter, sub-polar summer, sub-polar winter, 1976 US standard atmospheric profile), different aerosol models (villages, oceans, cities, troposphere, convective fog, radiation aerosol, etc.), different visibility, different water vapor content and different observation angle. Fig. 2 to Fig. 6 show the transmittance results of M12 and M13 channels of VIIRS that simulated by MODTRAN4 under different influence factors.

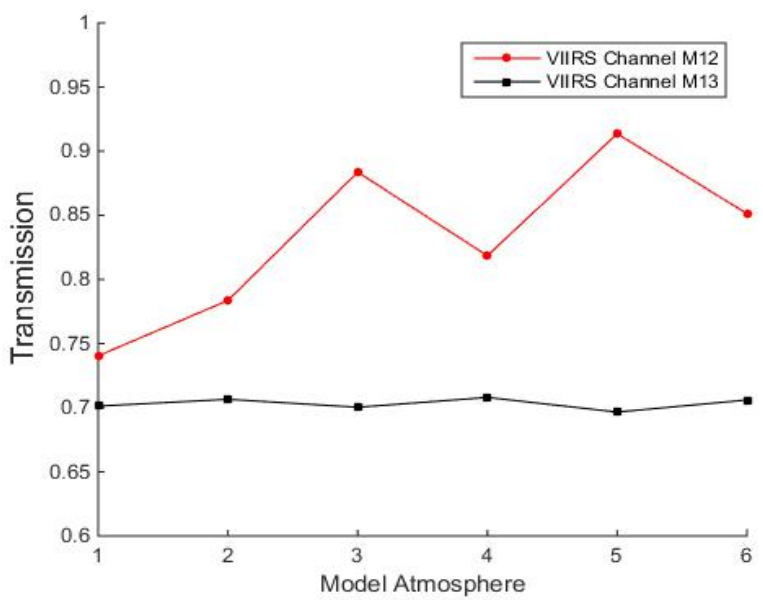

Fig.2 Atmospheric transmissions curve under 6 model atmospheres 


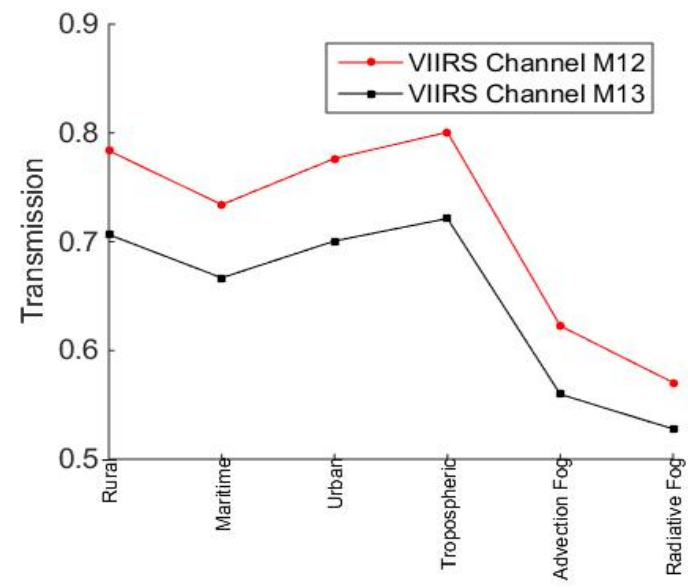

Fig.3 Atmospheric transmissions curve under 6 aerosol models
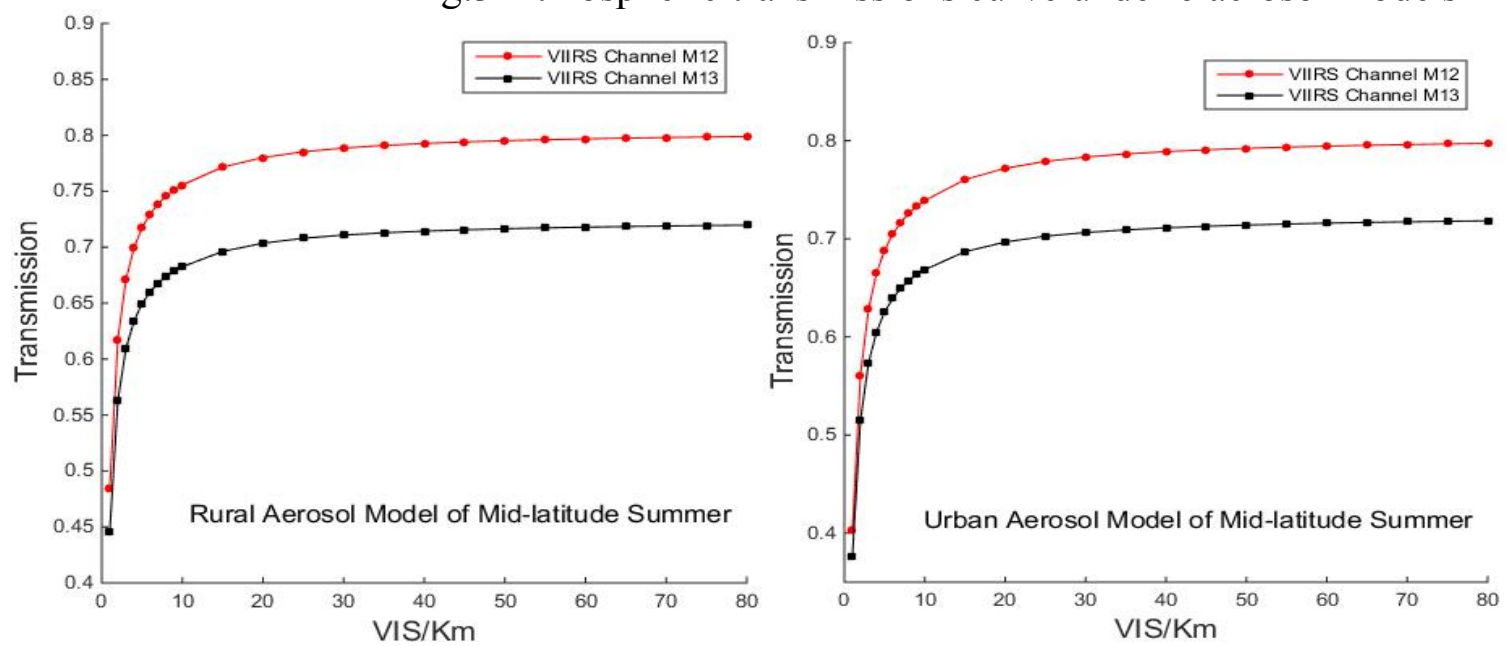

Fig.4 Atmospheric transmissions curve under different visibility in rural aerosol model and urban aerosol model 

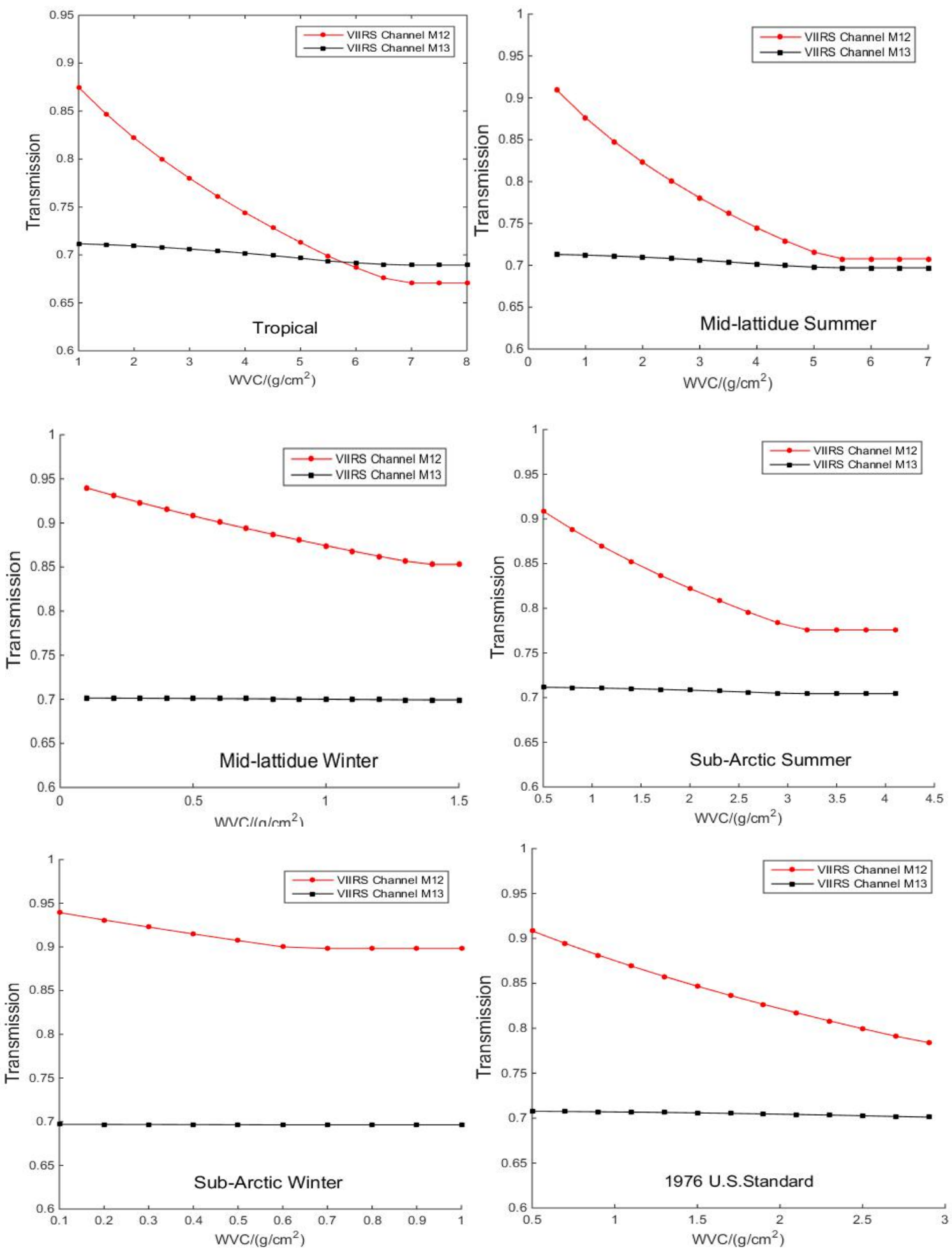

Fig.5 Atmospheric transmissions curve under different WVC in different atmosphere 


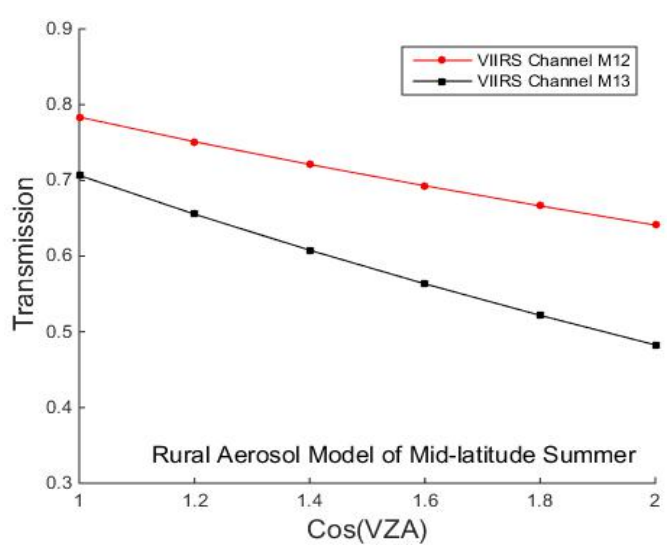

Fig.6 Atmospheric transmissions curve under different view angles

\section{Conclusions and discussion}

From the above simulation curves of channel transmittance, we can find that the transmittances of the both two middle infrared channels M12 and M13 of VIIRS are influenced by the aerosol type, visibility and observation angle. And the influence of these factors on the M12 channel transmittance are greater impact, in addition, M12 band is strongly influenced by the change of atmospheric profile. When the visibility is more than $15 \mathrm{Km}$, the channel transmittance of the two channels in the mid-latitude summer atmospheric profile is less affected by it. The atmospheric transmittance of the M13 channel almost does not change by the water vapor content, but the atmospheric transmittance of the M12 channel affected to a certain extent by it. Therefore, it is necessary to consider the influence of the change of water vapor content when using the M12 channel to invert other parameters (such as surface reflectivity, temperature, etc.). Through the simulation of channel transmittance of the M12 and M13 bands of VIIRS, it is possible to understand the factors that affect the atmospheric transmittance in the middle infrared band. So we can improve the accuracy of atmospheric correction and surface reflectivity inversion results, and on this basis, the inversion algorithm can also do further error analysis.

\section{Acknowledgements}

This work was financially supported by Doctoral Fund of Ministry of Education of China (20130001110046), the National Natural Science Foundation of China (41371492) and the Beijing Natural Foundation (Z151100000915068).

\section{References}

[1] Psiloglou B E, Santamouris M, Asimakopoulos D N: Renewable energy, 1995, 6(1): 63-70.

[2] Ruizhong Rao: Modern Atmospheric Optics (Science Press, 2012).

[3] Bohui Tang, Zhaoliang Li, Wu Hua, Tang Rong-Lin: The research about surface emissivity retrieval in thermal infrared (Science Press, 2014): 72-75.

[4] Zhiguo Rong, Yuxiang, Zhang, et al: Infrared \& Laser Engineering, 2009 38(4):589-593. [5] Yonggang Qian., Enyu Zhao, Caixia Gao, \& Ning Wang: IEEE Journal of Selected Topics in Applied Earth Observations \& Remote Sensing, 2014, 8(99):1208-1216.

[6] Jinhua Li, Zhibin Wang, Yuanyuan Chen: Laser \& Infrared, 2013, 43(10):1142-1145.

[7] Berk, A., Anderson, G. P., Acharya, P. K., Dothe, H., Adlergolden, S. M., \& Richtsmeier, S. C., et al.: The International Society for Optical Engineering, 1999,3756, 348-353.

[8] Arnone, R., Ladner, S., Fargion, G., \& Vandermeulen, R: The International Society for Optical Engineering, 2013, 8724(3), 363-383.

[9] Cao C, Xiong J, DeLuccia F, et al.: User's Guide, version, 2011, 1. 